\title{
IFN- $\gamma$ and Fas/FasL are required for the antitumor and antiangiogenic effects of IL-12/pulse IL-2 therapy
}

\author{
Jon M. Wigginton, ${ }^{1}$ Eilene Gruys, ${ }^{2}$ Lisa Geiselhart, ${ }^{2}$ Jeffrey Subleski, ${ }^{2}$ \\ Kristin L. Komschlies, ${ }^{3}$ Jong-Wook Park, ${ }^{2}$ Theresa A. Wiltrout, ${ }^{3}$ Kunio Nagashima, ${ }^{3}$ \\ Timothy C. Back, ${ }^{3}$ and Robert H. Wiltrout ${ }^{2}$ \\ ${ }^{1}$ Pediatric Oncology Branch, Center for Cancer Research, National Cancer Institute (NCI), Bethesda, Maryland, USA \\ ${ }^{2}$ Laboratory of Experimental Immunology, Center for Cancer Research, NCI at Frederick, Frederick, Maryland, USA \\ ${ }^{3}$ Intramural Research Support Program, Science Applications International Corporation-Frederick, Frederick, Maryland, USA \\ Address correspondence to: Robert H. Wiltrout, NCI at Frederick, Building 560, Room 31-93, Frederick, \\ Maryland 21702-1201, USA. Phone: (301) 846-1258; Fax: (301) 846-1673; E-mail: WiltroutR@ mail.ncifcrf.gov.
}

Received for publication June 6, 2000, and accepted in revised form May 14, 2001.

Systemic administration of IL-12 and intermittent doses of IL-2 induce complete regression of metastatic murine renal carcinoma. Here, we show that overt tumor regression induced by IL-12/pulse IL-2 is preceded by recruitment of $\mathrm{CD}^{+} \mathrm{T}$ cells, vascular injury, disrupted tumor neovascularization, and apoptosis of both endothelial and tumor cells. The IL-12/IL-2 combination synergistically enhances cell surface FasL expression on $\mathrm{CD}^{+} \mathrm{T}$ lymphocytes in vitro and induces Fas and FasL expression within tumors via an IFN- $\gamma$-dependent mechanism in vivo. This therapy also inhibits tumor neovascularization and induces tumor regression by mechanisms that depend critically on endogenous IFN- $\gamma$ production and an intact Fas/FasL pathway. The ability of IL-12/pulse IL-2 to induce rapid destruction of tumor-associated endothelial cells and regression of established metastatic tumors is ablated in mice with a dysregulated Fas/FasL pathway. The common, critical role for endogenous IFN- $\gamma$ and the Fas/FasL pathway in early antiangiogenic effects and in antitumor responses suggests that early, cytokine-driven innate immune mechanisms and $\mathrm{CD8}^{+} \mathrm{T}$ cell-mediated responses are interdependent. Definition of critical early molecular events engaged by IL-12/IL-2 may provide new perspective into optimal therapeutic engagement of a productive host-antitumor immune response.

J. Clin. Invest. 108:51-62 (2001). DOI:10.1172/JCI200110128

\section{Introduction}

As occurred with the clinical evolution of combination chemotherapy, recent studies have demonstrated that biologic agents with complementary molecular mechanisms of action can be used together to achieve synergistic biological and/or therapeutic antitumor activity. The mechanisms engaged in this setting may be complex, and definition of the relative role of these processes in mediating tumor regression may be necessary for maximizing the overall efficacy of biological therapy. IL-12 is an important immunoregulatory cytokine that enhances several functions of $\mathrm{T}$ and/or NK cells (1) and possesses potent IFN- $\gamma$-dependent therapeutic activity in various murine tumor models $(2,3)$. IL-12 can interact additively or even synergistically with IL-2, another potent antitumor cytokine, for enhancement of $\mathrm{T}$ and/or NK cell proliferation, cytokine production, and cytolytic activity $(1,4-6)$, as well as the production of nitric oxide by murine peritoneal macrophages (7). IL-12 and IL-2 can synergistically enhance the cytolytic activity of human PBMCs, tumor-infiltrating lymphocytes, or regional lymph node-derived lymphocytes against autologous tumor or tumor cell lines in vitro $(8,9)$, while systemic administration of low doses of IL-2 potentiates the ability of
IL-12-secreting fibroblasts to limit the growth of established pulmonary metastases of MC- 38 colon carcinoma (10). Conversely, systemic IL-12 administration enhances the antitumor effects of vaccination with IL-2-producing colon carcinoma (11) tumor cells.

We have reported previously that systemic administration of IL-12/pulse IL-2 can induce rapid and complete regression of well-established primary and/or metastatic murine renal carcinoma (Renca) (12) and spontaneous mammary carcinomas arising in transgenic mice (13). In particular, the ability of IL-12 and IL-2 to synergistically enhance IFN- $\gamma$ production $(3,14)$ and the central role of IFN- $\gamma$ in IL-12-mediated tumor responses $(1,3,15)$ suggested that IFN- $\gamma$ could also be an important component of IL-12/pulse IL-2-induced antitumor responses. In patients with metastatic melanoma or renal cell carcinoma, an initial increase, followed by a decrement in circulating IFN- $\gamma$ levels, may be observed in some patients treated with repeated doses of IL-12 alone, while sustained induction of IFN- $\gamma$ production and elevation of circulating IFN- $\gamma$ levels correlated with clinical responsiveness (16). Thus, an enhanced ability of IL-12/pulse IL-2 to induce IFN- $\gamma$ might keep levels of this cytokine above a critical threshold for tumor response. These findings are par- 
ticularly intriguing in that IFN- $\gamma$ alone has demonstrated limited efficacy in the clinical setting (17), suggesting that induction of endogenous IFN- $\gamma$ production by IL-12 may engage unique pathways and/or other cofactors in the local tumor microenvironment, which are critical to the overall biologic effects of IFN- $\gamma$. Therefore, the present studies were designed to identify pathophysiological events in the tumor microenvironment that mediate IL-12/pulse IL2 -induced antitumor mechanisms and have demonstrated a unique interrelationship between IFN- $\gamma$ and the Fas/FasL pathway in mediating vascular endothelial apoptosis, inhibition of tumor neovascularization, and overall tumor regression.

\section{Methods}

Mice and tumor cells. BALB/c mice were obtained from the Animal Production Area of the National Cancer Institute-Frederick Cancer Research and Development Center (Frederick, Maryland, USA). BALB/c-Ifng ${ }^{\text {tm1Ts }}$ (GKO) mice with targeted disruption of the IFN- $\gamma$ gene $\left(I F N-\gamma^{\prime-}\right)$ as well as C.B6-MRL $L^{\mathrm{lpr}}(\mathrm{lpr})$ and CPt.C3HFaslgld (gld) mice with mutated, nonfunctional Fas and FasL, respectively, were originally purchased from the Jackson Laboratory (Bar Harbor, Maine, USA) and then produced in our own colony. Mice were maintained in a dedicated pathogen-free environment and used between 8 and 12 weeks of age. Renca, a transplantable spontaneous murine renal carcinoma of $\mathrm{BALB} / \mathrm{c}$ origin (18), was used for all experiments.

Reagents. Recombinant murine IL-12 was generously provided by the Genetics Institute Inc. (Cambridge, Massachusetts, USA) and Hoffmann LaRoche Inc. (Nutley, New Jersey, USA). For in vivo drug administration, stock aliquots were diluted with PBS containing $0.1 \%$ (vol./vol.) sterile-filtered BALB/c mouse serum. Highly purified, recombinant human IL-2 (from Escherichia coli) was generously provided by Chiron Corp. (Emeryville, California, USA) $(19,20)$. After reconstitution with sterile water, IL-2 was diluted with HBSS containing $0.1 \% \mathrm{BALB} / \mathrm{c}$ mouse serum for in vivo administration.

Tumor models. Subcutaneous primary Renca tumors were established by midflank injections of $10^{5}$ tumor cells in $0.2 \mathrm{ml} \mathrm{HBSS}$, while orthotopic tumors were established by intrarenal injection of $10^{5}$ tumor cells (12). Where indicated, the tumor-bearing kidney was surgically resected 11-13 days after tumor cell implantation as described previously (12). By this time, widespread distant metastases (lung and liver) have uniformly occurred in all mice. In some studies, nephrectomy of the tumor-bearing kidney was not performed, and therapy was administered as indicated to mice bearing established intrarenal primary tumors.

In vivo treatment regimens. In general, treatment of Renca-bearing mice (10 mice per group) was initiated 7-11 (subcutaneous implants) or 8-13 (intrarenal implants) days after tumor implantation. The IL$12 /$ pulse IL-2 regimen consisted of the twice-daily intraperitoneal injection of 300,000 IU IL-2 plus one intraperitoneal injection of $0.5 \mu \mathrm{g}$ IL-12 or vehicle alone on the first day, followed by injection of $0.5 \mu \mathrm{g}$ IL-12 or vehicle alone on the next 3-4 days. Mice were then routinely rested for 2-3 days between each cycle and received one to four cycles of therapy, depending on the nature of the experimental question. The exact regimens used for each study are described in the appropriate figure legends.

Cytokine production and assays. Normal control mice as well as tumor-bearing mice were treated with two cycles of IL-12 with or without pulse IL-2 or vehicle alone. The mice were then euthanized and single cell suspensions of murine splenocytes were prepared in complete medium consisting of RPMI-1640 with $10 \%$ FBS (Intergen Co., Purchase, New York, USA) and additives, including $1 \%$ sodium pyruvate, $1 \%$ L-glutamine, $1 \%$ penicillin/streptomycin, and $1 \%$ nonessential amino acids (BioWhittaker Inc., Walkersville, Maryland, USA). Cells were aliquoted in duplicate in 24-well flat-bottom plates (Corning-Costar Corp., Cambridge, Massachusetts, USA) at $10^{6}$ cells $/ \mathrm{ml}$ in complete medium containing hamster anti-mouse CD3 (2C11) and hamster anti-mouse CD28 (50 ng/ml; PharMingen, San Diego, California, USA) or medium alone. Cells were incubated for 48 hours, and supernatants were subsequently assayed for IFN- $\gamma$ by ELISA using matched Ab pairs (PharMingen). Serum samples from these mice treated with IL-12/pulse IL-2 or vehicle alone were assayed as well for IFN- $\gamma$ using ELISA.

Intracellular cytokine analysis. $\mathrm{BALB} / \mathrm{c}$ mice were treated with HBSS or IL-12 $(0.5 \mu \mathrm{g}$ daily on days $0-2)$ and IL-2 (300,000 IU twice daily on day 0$)$. On day 3 , mice were euthanized, spleens were removed, and splenocytes were recovered by disrupting spleens in the presence of RPMI-1640 brefeldin medium containing 5 $\mu \mathrm{g} / \mathrm{ml}$ brefeldin A (Sigma Chemical Co., St. Louis, Missouri, USA), $5 \% \mathrm{FBS}, 2 \mathrm{mM}$-glutamine, $1 \mathrm{mM}$ sodium pyruvate, and $5 \times 10^{-5} \mathrm{M} 2$-mercaptoethanol (M2-ME). After incubating splenocytes for 4 hours in RPMI-1640 brefeldin medium in a $37^{\circ} \mathrm{C} \mathrm{CO}^{2}$ incubator, IFN- $\gamma$ intracellular labeling was performed using the Cytofix/Cytoperm kit (PharMingen), according to the manufacturer's suggested protocol using optimally titered anti-mouse IFN- $\gamma$ (clone XMG1.2; PharMingen). Cells (10,000 per sample) were analyzed for the percentage of cells expressing mouse IFN- $\gamma$ by using a FACScan flow cytometer affixed with a 488-nm laser (Becton Dickinson Immunocytometry Systems, Mountain View, California, USA). Absolute numbers of cell populations expressing IFN- $\gamma$ were determined by multiplying these percentages by the total splenic cellularity. Isotype-matched controls were used to quantitate nonspecific background binding that was subtracted from all results.

Immunohistochemistry. Subcutaneous Renca tumor implants from mice treated with two cycles of either IL-12/pulse IL-2 or vehicle alone were embedded in Tissue Tek OCT medium, frozen on dry ice, and stored 
below $-70^{\circ} \mathrm{C}$ until further use. Cryopreserved tissues were sectioned at $5 \mu \mathrm{M}$ and stained for the presence of $\mathrm{CD}^{+}$or $\mathrm{CD}^{+} \mathrm{T}$ cells using rat anti-mouse CD4 and rat anti-mouse CD8 primary Ab's (PharMingen) and standard immunohistochemical techniques. Established intrarenal Renca tumor implants treated with either IL-12/pulse IL-2 or vehicle alone for one and one-half cycles were processed in a similar fashion and stained for the vascular marker CD31 using the rat anti-mouse CD31 (clone 390) primary Ab (PharMingen) and standard immunohistochemical techniques.

$R T-P C R$. Total cellular RNA was isolated from snapfrozen tissue specimens by the Trizol method. Reverse transcription and PCR reactions were performed consistent with methods we have described previously $(13,21)$.

The sequence of primer pairs and the predicted product size for the respective genes evaluated in these studies are as follows: GAPDH: sense GCCACCCAGAAGACTGTGGATGGC, antisense CATGTAGGCCATGAGGTCCACCAC; IP-10: sense ACCATGAACCCAAGTGCTGCCGTC, antisense GCTTCACTCCAGTTAAGGAGCCCT; Mig: sense GATCAAACCTGCCTAGATCC, antisense GGCTGTGTAGAACACAGAGT; Fas: sense CTTCTACTGCGATTCTCCTGGCT, antisense TCTCCTCTCTTCATGGCTGGAAC; FasL: sense CAGCTCTTCCACCTGCAGAAGG, antisense AGATTCCTCAAAATTGATCAGAGAGAG.

Induction of lymphocyte FasL expression. Single cell suspensions of normal BALB/c lymph node-derived lymphocytes were prepared in complete medium as described above, also containing $50 \mu \mathrm{M} 2$-mercaptoethanol (Sigma Chemical Co.). The lymphocytes were then seeded into T75 flasks at a final concentration of $2 \times 10^{6}$ cells $/ \mathrm{ml}$ in complete media containing $5 \mu \mathrm{g} / \mathrm{ml}$ concanavalin A (Sigma Chemical Co.) for 2 days, and then the nonadherent cells were decanted, washed with HBSS, and reseeded in $50 \mathrm{ml}$ of supplemented RPMI containing $10 \%$ FBS and $1,200 \mathrm{IU} / \mathrm{ml}$ IL-2. Cells were fed with an additional $100 \mathrm{ml}$ of complete medium on days 3 and 4 and harvested on day 5 . The cells were then washed twice with HBSS and seeded into six-well flat-bottom plates (Corning-Costar Corp.) at a final concentration of $10^{6}$ cells $/ \mathrm{ml}$ in complete media containing $10 \mu \mathrm{M}$ freshly prepared KB8301 metalloproteinase inhibitor (PharMingen) to prevent proteolysis of FasL in vitro. IL-12 $(50 \mathrm{ng} / \mathrm{ml}$ final concentration) and/or IL-2 (150 IU/ml [25 cetus units $/ \mathrm{ml}$ ] final concentration, as indicated) or medium alone were added as appropriate. Twenty-four hours after culture initiation, the cells were harvested, washed twice in HBSS, and then plated at $10^{6}$ cells per well in 96-well round-bottom plates (Corning-Costar Corp.). The cells were then preincubated with an excess of the monoclonal rat anti-mouse CD16/32 Ab (clone 2.4G2) (PharMingen) to block cell surface Fc receptors and prevent nonspecific $\mathrm{Ab}$ binding. Cell surface expression of the CD8 and CD4 T lymphocyte subset markers was determined by staining with purified, FITC-labeled monoclonal rat anti-mouse CD8a (clone 53-6.7) or rat anti-mouse CD4 (clone GK1.5)
Ab's (PharMingen). FITC-labeled rat IgG2a (clone R35-95) (PharMingen) was used as an isotype control. Cell surface FasL expression was determined using the three-step system recommended by PharMingen. Briefly, cells were stained with hamster anti-mouse FasL (clone MFL3) (PharMingen) or hamster IgG (clone A19-3) isotype control Ab's (PharMingen) followed by incubation with a cocktail of biotin-conjugated mouse anti-hamster IgG Ab's (clones G70-204 and G94-56) (PharMingen) and then streptavidin-phycoerythrin (PE) (PharMingen). Each step included an incubation for 15 minutes at $4^{\circ} \mathrm{C}$ and then a wash with HBSS containing $0.1 \%$ BSA and sodium azide (Sigma Chemical Co.). After staining, cell surface expression of the respective markers (i.e., CD4, CD8, FasL, etc.) was detected and quantified on 10,000 total cells using a FACScan (Becton Dickinson Immunocytometry Systems, San Jose, California, USA) and CelQQuest analysis software.

Electron microscopy. Renal tumors were initially fixed in $4 \%$ paraformaldehyde and 2\% glutaraldehyde in PBS (Tousimis Research Corp. Inc., Rockville, Maryland, USA), trimmed into $3-4 \mathrm{~mm}^{3}$ pieces with a singleedged razor blade, post fixed in 1\% osmium (Stevens Metallurgical Corp., New York, New York, USA), and embedded in epoxy resin LX-110 (Ladd Research Industries Inc., Burlington, Vermont, USA). The embedded tissue was then sectioned to approximately 50-60 nm and mounted on a copper-mesh grid. The thin sections were stained in uranyl acetate and lead citrate solution to enhance the contrast, stabilized by carbon evaporation in a vacuum evaporator (Denton Vacuum Inc., Moorestown, New Jersey, USA), and examined and photographed with an H7000 electron microscope (Hitachi Ltd., Tokyo, Japan).

Latex infusion assays of neovascularization. To visualize and quantitate changes in tumor vascularity, Rencabearing mice were euthanized, tails were snipped at the base to provide an open circulatory circuit below the diaphragm, and $3 \mathrm{ml}$ of $50 \%$ latex suspension (Carolina Biological Supply Co., Burlington, North Carolina, USA) in PBS was infused intravenously into each mouse via the retro-orbital plexus. After 10 minutes to allow the latex to solidify, the vascular supply leading to the tumor-bearing kidney was ligated, and the tumor-bearing kidney was resected en bloc and placed in $10 \%$ neutral buffered formalin. For direct quantitation of overall tumor vascularity, ${ }^{111}$ indium-oxine (111In-ox; Medi-Physics Inc., Arlington Heights, Illinois, USA) was added to the latex suspension at a final concentration of $3 \times 10^{5} \mu \mathrm{Ci} / \mathrm{ml}$. This mixture was then infused, and the tumor-bearing kidney and contralateral normal kidney were resected, placed individually in vials, and the total amount of radioactivity within each organ was counted.

A direct correlation of infused radioisotope-latex with increased vascularity, which was independent of tumor size, was confirmed by the adaptation of a model of neovascularization described previously (22). 


\section{Figure 1}

Infiltration of $\mathrm{CD}^{+} \mathrm{T}$ lymphocytes in established Renca tumors after treatment with IL-12/pulse IL-2. Cohorts of mice received subcutaneous Renca implants and were subsequently treated with IL-12 ( $0.5 \mu \mathrm{g}$ daily on days $11-15$ and $18-21$ ), pulse IL-2 (300,000 IU twice daily on days 11 and 18), or vehicle alone. After two cycles of therapy, tumors were resected and stained for expression of CD8 or CD4. Anti-CD8 sections from control mice (c) and mice treated with IL-12/pulse IL-2 (d) and antiCD4-stained sections from control (a) and IL-12/pulse IL-2-treated (b) mice are shown. $\times 200$.

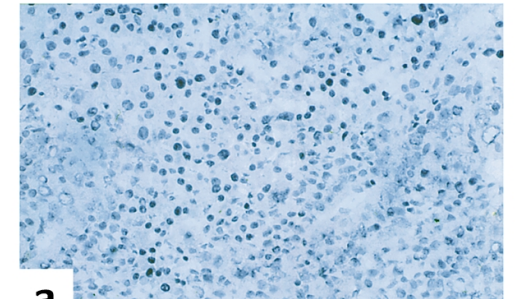

a
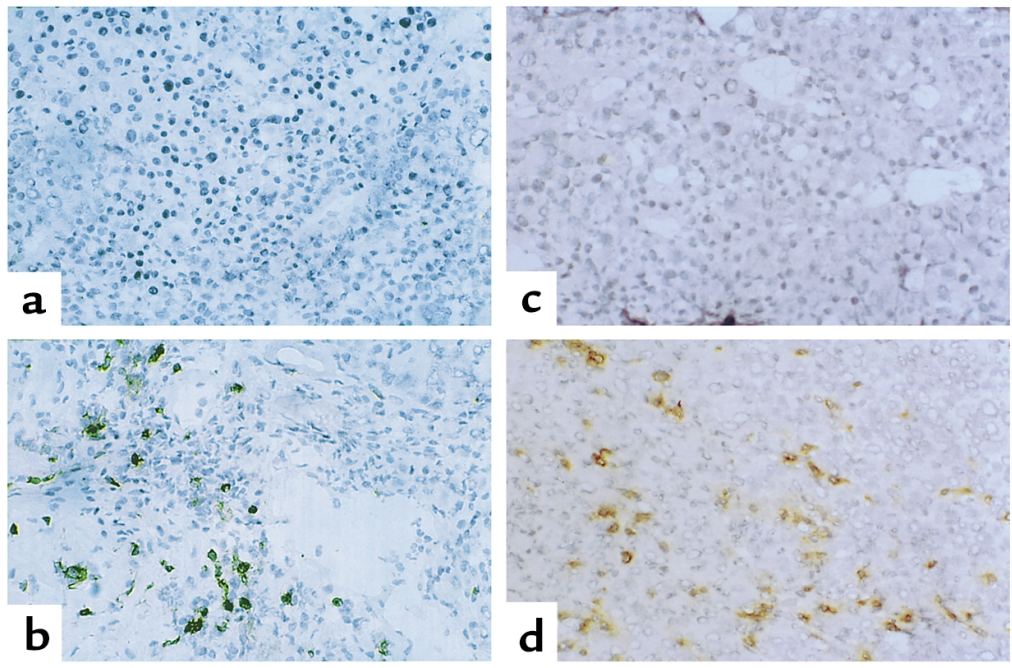

For these studies, sterile Gelfoam sponges (Pharmacia and Upjohn Co., Kalamazoo, Michigan, USA) were cut into squares of approximately $5 \times 5 \times 5 \mathrm{~mm}\left(125 \mathrm{~mm}^{3}\right)$, hydrated, and deaerated in sterile PBS overnight. The sponges were then blotted dry and equilibrated in 50 $\mathrm{mmol} / \mathrm{l}$ Tris- $\mathrm{HCl}, \mathrm{pH} 7.4$, for 60 minutes at $37^{\circ} \mathrm{C}$. After equilibration, the sponges were again blotted dry and incubated for 60 minutes at $37^{\circ} \mathrm{C}$ with $85 \mu$ of 50 $\mathrm{mmol} / \mathrm{l}$ Tris/ $\mathrm{HCl}$ ( $\mathrm{pH} 7.4$ ) containing $1.25 \mu \mathrm{g}$ of bFGF. Mice were anesthetized, their abdomens washed with $70 \%$ ethanol, and an incision made along the midline to expose the abdominal cavity. Small incisions were made on a limited area of the capsule on the left kidney, and control- or bFGF-infused sponges were affixed by cyanoacrylate adhesive. The margins of the surgical incision were closed with 9-mm autoclips. Ten days later, mice were euthanized and infused with ${ }^{111} \mathrm{In}$ ox-containing latex as described above. Sponges were then removed, and the amount of radioactivity in each sponge was quantitated.
Statistical methods. Data in this study were evaluated and reported using descriptive techniques, the Fisher exact test, as well as standard two-sample $t$ tests. The proportions of mice achieving complete tumor regression were compared using the Fisher exact test. The absolute number of counts of radioactivity in the tumor-bearing kidneys or Gelfoam sponges and the ratios of radioactivity counts in the tumor-bearing kidney/non-tumor-bearing kidney among mice treated with IL-12/pulse IL-2 were compared with those among control mice treated with vehicle using the standard two-sample $t$ test. Probability values were obtained from two-tailed tests and were considered significant when $P$ values were less than 0.05 .

\section{Results}

Accumulation of $C D 8^{+} T$ lymphocytes in regressing tumors after administration of IL-12/pulse IL-2. Previous studies from our laboratory have implicated $\mathrm{T}$ cells in the therapeutic activity of this combination (12), and using

\footnotetext{
Figure 2

Detection of intracellular IFN- $\gamma$ in splenic T lymphocyte subsets after in vivo treatment with IL12/pulse IL-2. BALB/c mice were treated with IL-12 ( $0.5 \mu \mathrm{g}$ daily on days 0,1 , and 2 ) and pulse IL-2 $(300,000$ IU twice daily on day 0$)$ or vehicle alone. On day 3 , single cell suspensions of splenic leukocytes were prepared, and intracellular IFN- $\gamma$ expression by $\mathrm{CD}^{+}$and $\mathrm{CD} 8^{+} \mathrm{T}$ lymphocytes was determined by flow cytometry as described in Methods. Histograms represent the percentages of $\mathrm{CD}^{+}$and $\mathrm{CD} 8{ }^{+} \mathrm{T}$ cells, respectively, which express IFN- $\gamma$ after treatment with IL-12/pulse IL-2 or vehicle alone. Bar graphs represent the absolute number of cells expressing IFN- $\gamma$ per spleen $\left(\times 10^{5}\right)$.
}

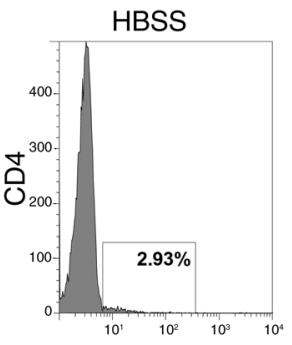

IL-12/IL-2

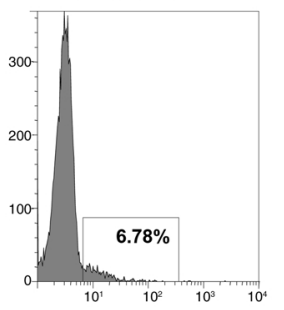

IFN- $\gamma$
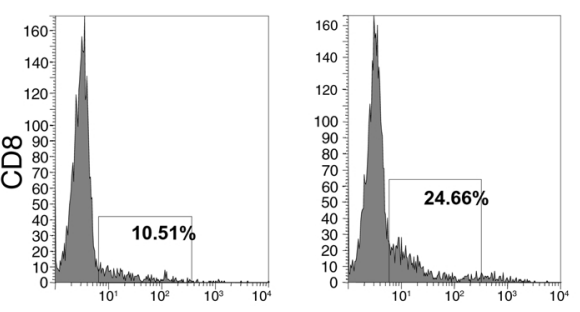
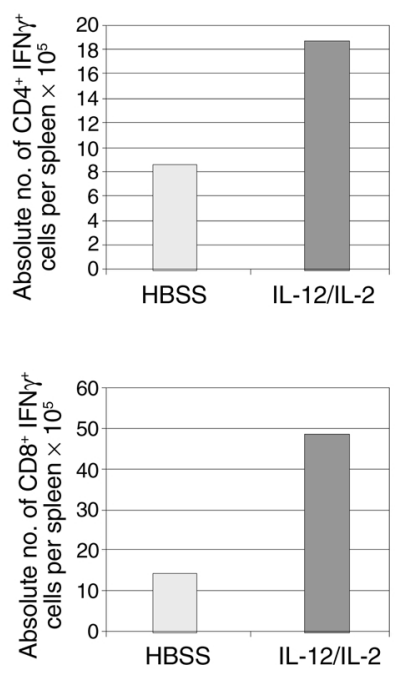


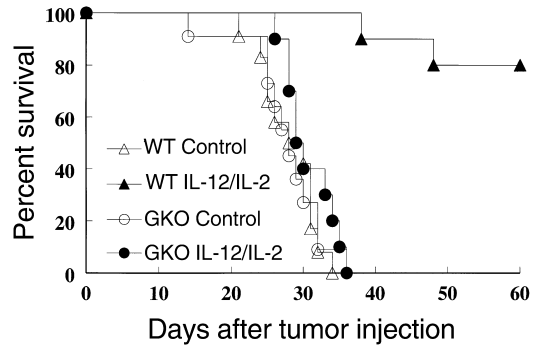

Figure 3

Role of IFN- $\gamma$ in the antitumor activity of IL-12/pulse IL-2 against metastatic Renca. WT versus IFN- $\gamma^{-1-}$ mice received intrarenal Renca tumor implants. Mice underwent unilateral nephrectomy to remove the primary tumor-bearing kidney on day 12 after tumor implantation and were subsequently treated with IL-12 (0.5 $\mu \mathrm{g}$ daily on days 13-17, 20-24, 27-31, and 34-38), pulse IL-2 (300,000 IU twice daily on days $13,20,27,34$, and 41 ), or vehicle alone. Mice surviving at last follow-up were tumor-free.

depletion studies, we have subsequently confirmed that $\mathrm{CD}^{+}$but not $\mathrm{CD}^{+}$effector cells are vital for the IL-12/pulse IL-2-induced response (data not shown). These results suggested that recruitment of $\mathrm{CD}^{+} \mathrm{T}$ cells into tumor sites would be required for IL-12/pulse IL-2-induced response. In fact, substantial increases in the infiltration of $\mathrm{CD}^{+} \mathrm{T}$ cells into subcutaneous Renca tumors were noted in treated mice (Figure 1d) compared with tumor sections from control mice treated with vehicle alone (Figure 1c). Similar increases in local infiltration of noncritical $\mathrm{CD} 4^{+} \mathrm{T}$ cells were also noted in tumors from mice treated with IL-12/pulse IL-2 (Figure 1b) compared with control mice treated with vehicle alone (Figure 1a).

Role of endogenous IFN- $\gamma$ in the antitumor activity of IL-12/pulse IL-2. The implication of induced IFN- $\gamma$ in IL12 -mediated antitumor effects in mice $(3,15)$ and the association of sustained IFN- $\gamma$ production with clinical responses observed in humans treated with IL-12 alone (16) suggest that enhanced induction of IFN- $\gamma$ by IL12 pulse IL-2 also could contribute to the antitumor effects of this combination. The ability of murine splenocytes to produce IFN- $\gamma$ upon subsequent activa- tion ex vivo with anti-CD3/anti-CD28 was substantially increased in mice treated previously in vivo with IL-12/pulse IL-2 (93 pg/ml) compared with IL-12 (55 $\mathrm{pg} / \mathrm{ml})$, IL-2 $(26 \mathrm{pg} / \mathrm{ml})$, or vehicle $(22 \mathrm{pg} / \mathrm{ml})$ alone (data not shown). We also have detected marked increases in serum IFN- $\gamma$ levels in tumor-bearing mice treated with IL-12/pulse IL-2 (1,535 pg/ml) versus control mice treated with vehicle alone (undetectable, less than $50 \mathrm{ng} / \mathrm{ml}$ )(data not shown). Analysis of the early cellular sources for IFN- $\gamma$ revealed that by 3 days after the administration of IL-12/pulse IL-2 there were twofold to threefold increases in the percentages and total number of both splenic $\mathrm{CD}^{+}$and $\mathrm{CD}^{+} \mathrm{T}$ cells expressing IFN- $\gamma$ (Figure 2). However, the total number of $\mathrm{CD}^{+} \mathrm{T}$ cells positive for intracellular IFN- $\gamma$ was approximately threefold greater than the number of $\mathrm{CD} 4^{+} \mathrm{T}$ cells. These findings further suggest a potentially important role for IFN- $\gamma$ in the antitumor activity of IL-12/pulse IL-2. This hypothesis was confirmed in wild-type (WT) versus $I F N-\gamma^{\prime-}$ mice bearing established metastatic Renca, where the therapeutic activity of IL-12/pulse IL-2 seen in WT mice was completely lost in IFN- $\gamma^{-1-}$ mice (Figure 3). Complete responses were induced in $70 \%$ (seven of ten) of WT mice versus $0 \%$ (zero of ten) of $I F N-\gamma^{-1}$ mice treated with IL$12 /$ pulse IL-2 $(P=0.0007)$, thus demonstrating that endogenous IFN- $\gamma$ is a central mediator of the overall antitumor activity of IL-12/pulse IL-2.

Histopathological analyses of the antitumor effects of IL12/pulse IL-2: role of IFN- $\gamma$. The potent antitumor responses described above and the central role of IFN- $\gamma$ suggested an important contribution by IFN- $\gamma$-dependent mechanisms in the local tumor microenvironment. Hematoxylin and eosin-stained Renca tumor sections from mice treated with IL-12/pulse IL-2 demonstrate substantial reductions in vascularity and large areas of apoptosis/coagulative necrosis compared with tumors from mice treated with vehicle alone (data not shown). The role of IFN- $\gamma$ in mediating the antineovascular effects of IL-12/pulse IL-2 was subsequently evaluated in tumors stained with the vascular marker, CD31. Established tumors from WT or IFN- $\gamma^{-1}$ mice treated

\section{Figure 4}

Role of IFN- $\gamma$ in the antivascular activity of IL-12/pulse IL-2 against intrarenal Renca. WT versus IFN- $\gamma^{--}$mice received intrarenal Renca tumor implants. Mice were treated with IL-12 (0.5 $\mu \mathrm{g}$ daily on days $11-15$ and $18-20)$, pulse IL-2 (300,000 IU twice daily on days 11 and 18), or vehicle alone. Tumors were resected on day 21 and stained for CD31 expression. Representative sections from WT mice treated with IL-12/pulse IL-2 (b) or vehicle alone (a) and IFN- $\gamma^{-/}$mice treated with IL-12/pulse IL-2 (d) or vehicle alone $(\mathbf{c})$ are shown. $\times 100$.
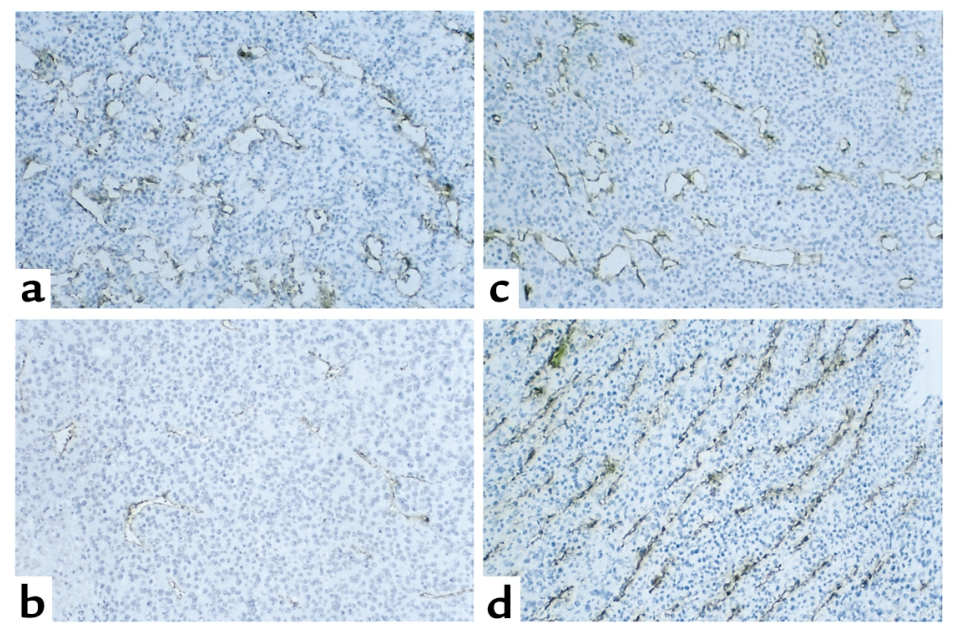


\section{Figure 5}

Vascularity of advanced intrarenal Renca tumors and impact of IL-12/pulse IL-2 administration on tumor neovascularization. (a-c) The extensive vascularity of untreated advanced ( 3.5 weeks old) intrarenal Renca tumors was visualized as described in Methods. Using these methods, the vascularity of established intrarenal Renca tumors was compared directly after treatment of mice with IL-12 ( $0.5 \mu$ g daily on days $8-12$ and $15-18)$ plus pulse IL-2 (300,000 IU twice daily on days 8 and 15) (f) or vehicle alone (d and e). Quantitative assessment of the impact of IL-12/pulse IL-2 administration on vascularization of intrarenal Renca tumor implants $(\mathbf{g})$. Cohorts of mice received subcapsular intrarenal Renca tumor implants and were treated with IL-12 (0.5 $\mu$ g on days $12-16$ and $19-22)$, pulse IL-2 (300,000 IU twice daily on days 12 and 19), or vehicle alone. At baseline (day 12) and after one (day 16) or two (day 22) cycles of therapy, cohorts of mice were euthanized, and latex suspension containing ${ }^{111}$ In-ox was infused as described in Methods. The normal and tumor-bearing kidneys were resected, and the amount of radioactivity in each organ was quantitated. Values for the tumorbearing kidney were normalized to the non-tumor-bearing kidney in each animal. Open circles represent the respective values for individual mice euthanized at the indicated time point.

with vehicle alone grew progressively and were highly vascularized (Figure 4, a and c, respectively), while administration of IL-12/pulse IL-2 to WT (Figure 4b) but not $I F N-\gamma^{\prime-}$ (Figure 4d) mice resulted in clear decreases in overall tumor vascularity, demonstrating a central role for IFN- $\gamma$ in both antineovascular and antitumor effects of this regimen.

Administration of IL-12/pulse IL-2 inhibits tumor neovascularization. The dramatic histopathological changes described above suggest that the antitumor effects induced by IL-12/pulse IL-2 may be related to an overall antineovascular effect and/or to direct destruction of tumor cells. To more definitively examine potential antineovascular effects in vivo directly within the actual tumor site, we developed a novel method using latex infusion to visualize directly the tumor vascular supply and detect changes in overall vascularization that were induced by IL-12/pulse IL-2. As shown in Figure 5 , a-c, intrarenal Renca tumor implants form large, highly vascularized tumors. Using the latex-infusion
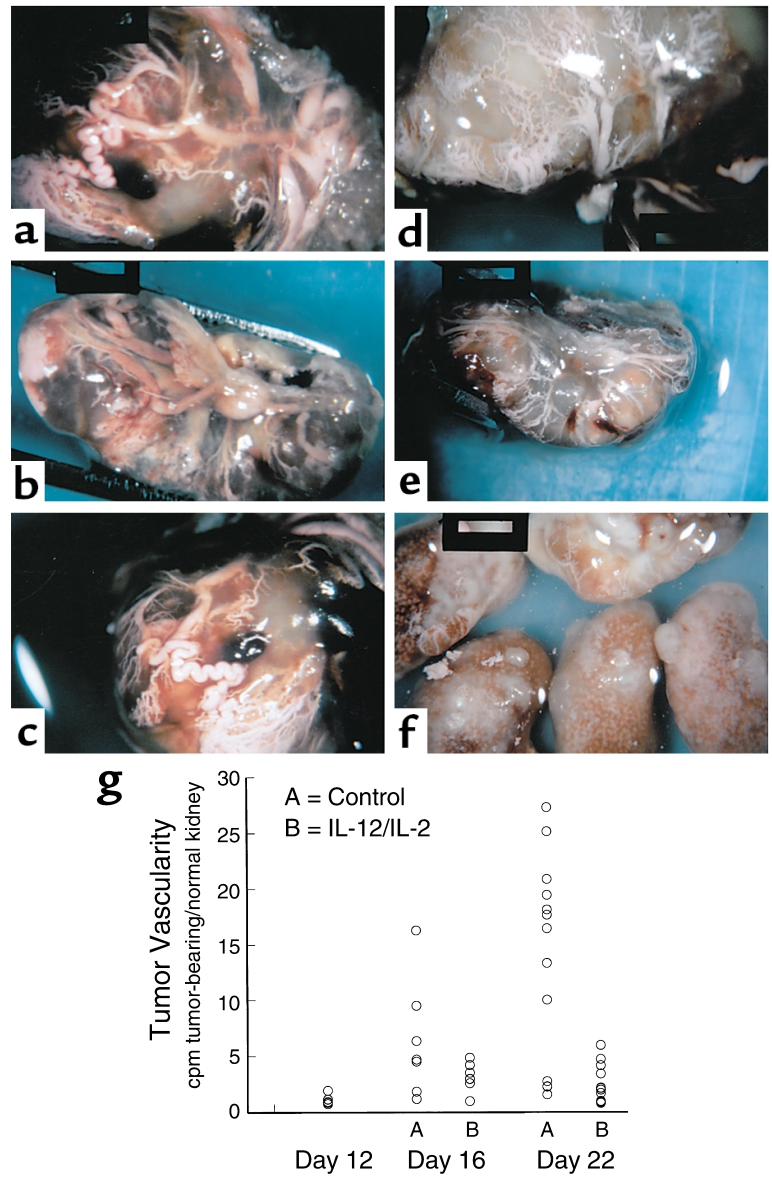

Day of treatment

method, striking decreases in tumor size and overall vascularity were observed in intrarenal tumor implants after two cycles of therapy with IL-12/pulse IL-2 (i.e., 19 days after tumor implantation) (Figure 5f) when compared with implants from mice treated with vehicle alone (Figure 5, d and e).

This effect was directly quantitated by incorporating ${ }^{111}$ In-ox into the latex infusate and counting the amount of radioactivity in both the tumor-bearing and non-tumor-bearing kidneys. The data are presented as a normalized index of overall tumor vascularization by
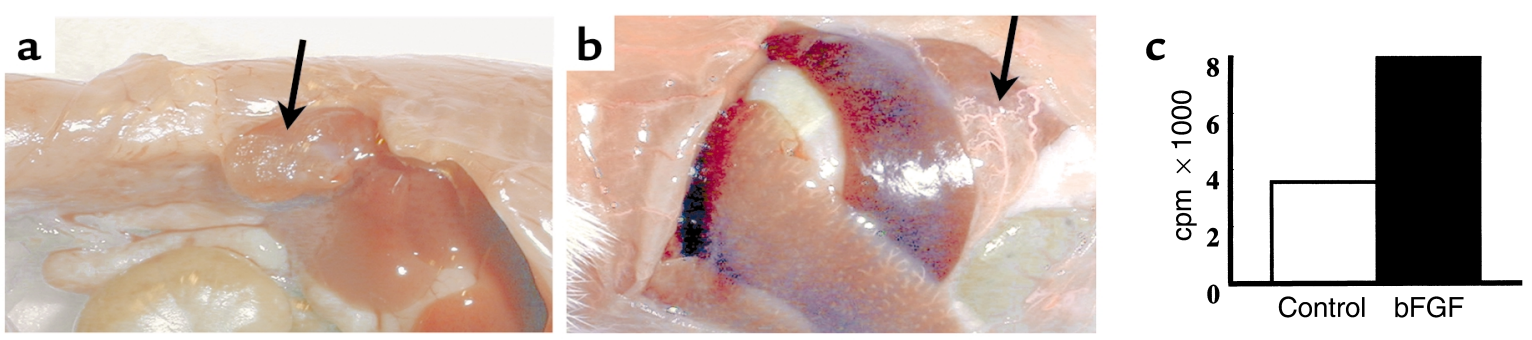

Figure 6

Demonstration of direct correlation between increased accumulation of ${ }^{111} \mathrm{In}$-ox latex infusate and increased vascularity of Gelfoam sponges (control or bFGF-treated) were affixed onto the surface of the left kidney as described previously. Ten days later, mice were euthanized, infused with ${ }^{111} \mathrm{In}$-ox-containing liquid latex as described for Figure 5, and sponges were excised for visual inspection and quantitation of radioisotope incorporation. The results show that as expected, control sponges are poorly vascularized (a), bFGF sponges are well vascularized (b), and the degree of vascularity correlates directly with an increase in isotope accumulation (c). 


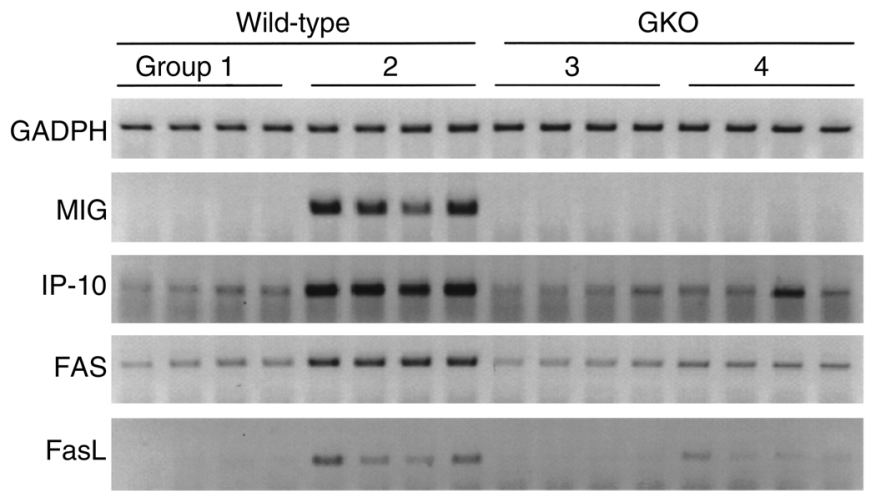

dividing the counts per minute in the tumor-bearing kidney by the counts per minute in the non-tumorbearing kidney in each mouse. Using this approach, the vascularity of intrarenal Renca tumors was assessed at baseline before the initiation of therapy (day 12) and after the completion of one (day 16) or two (day 22) cycles of treatment with IL-12/pulse IL-2 or vehicle alone (Figure $5 \mathrm{~g}$ ). After one cycle of therapy (day 16), there was a trend toward a decrease in the absolute number of counts per minute per tumor-bearing kidney among mice treated with IL-12/pulse IL-2 (mean counts per minute $=49,622$ ) versus vehicle alone (mean counts per minute $=57,880$ ) and in the vascular indices of mice treated with IL-12/pulse IL-2 (mean index $=3.2$ ) versus vehicle alone (mean index $=6.3$ ). After two cycles of therapy (day 22), this trend evolved into a highly significant reduction $(P=0.0051)$ in the absolute number of counts per minute per tumor-bearing kidney in mice treated with IL-12/pulse IL-2 (mean counts per minute $=33,369$ ) versus vehicle alone (mean counts per minute $=94,495$ ) and in the vascular indices $(P=0.0001)$ for mice treated with IL-12/pulse IL-2 (2.3) versus vehicle alone (13.6). Collectively, these observations demonstrate that administration of IL-12/pulse IL-2 inhibits the progressive neovascularization of intrarenal Renca tumor implants.

To confirm that the increased incorporation of ${ }^{111}$ In-ox radioisotope in vascularized tumors was not simply a reflection of tumor size, we implanted identically sized control and bFGF-infused Gelfoam sponges onto the surface of the left kidney, and 10 days later infused these mice with ${ }^{111}$ In-ox latex as above. This approach results in minimal vascularization of the control sponges (Figure $6 a)$ and increased vascularity of the bFGF sponges (Figure 6b) (22). These differences correlated directly with increased radioisotope accumulation in the bFGF-infused sponges (Figure $6 \mathrm{c}$ ). This finding supports the conclusion that changes in ${ }^{111}$ In-ox con-

\section{Figure 7}

Impact of IL-12/pulse IL-2 administration on local gene expression within established Renca tumors in WT versus IFN- $\gamma^{/-}$mice. Subcutaneous Renca tumors were resected from mice treated with IL-12 ( $0.5 \mu \mathrm{g}$ daily on days $11-15$ and $18-20)$, pulse IL-2 (300,000 IU twice daily on days 11 and 18), or vehicle alone, and gene expression was characterized by RT-PCR. Each lane represents analysis of material from an individual tumor site.

tent in control or IL-12/IL-2-treated tumor-bearing mice relate directly to changes in the overall vasculature of the tumors.

IFN- $\gamma$-dependent induction of antiangiogenic and proapoptotic gene expression in the tumor microenvironment by administration of IL-12/pulse IL-2. The data described above demonstrate that the overall antitumor activity, as well as the potent anti-neovascular effects induced in the local tumor site by IL-12/pulse IL-2, are critically dependent on IFN- $\gamma$. Therefore, we investigated the ability of IL-12/pulse IL-2 to alter expression of genes encoding various modulators of angiogenesis (i.e., the angiogenic phenotype) as well as potential mediators of endothelial and/or tumor cell apoptosis within primary Renca tumors growing in WT versus IFN- $\gamma^{-1-}$ mice. Administration of IL-12/pulse IL-2 potently induced expression of the genes encoding IFN- $\gamma$ (not shown), as well as the antiangiogenic chemokines IP-10 and MIG and Fas and FasL, two
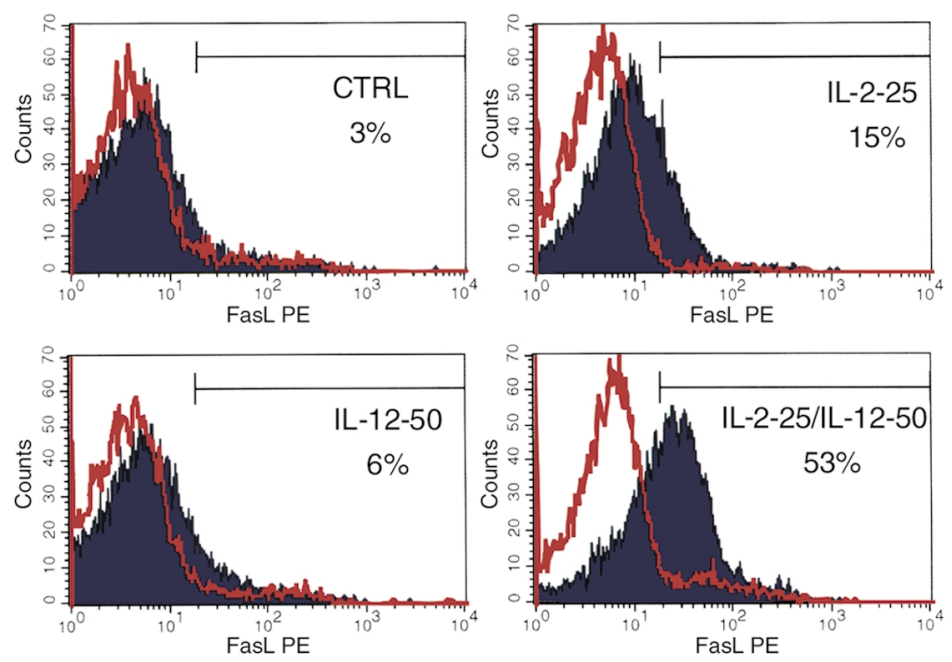

Figure 8

Impact of IL-12 with or without IL-2 on cell surface FasL expression by lymph node-derived lymphocytes. Activated lymph node-derived whole lymphocyte populations were cultured in vitro in the presence of KB8301 metalloproteinase inhibitor with IL-12, IL-2, or medium alone, and cell surface FasL expression was subsequently detected using immunofluorescent labeling with flow cytometric analysis as described in Methods. The respective histograms represent the fluorescent intensity distribution for cells cultured under the indicated conditions. The red and blue tracings represent fluorescence-intensity distribution histograms for cells cultured under the indicated conditions and stained subsequently with isotype control or hamster anti-mouse FasL Ab's, respectively. The percentage of cells that were positive for Fas $L$ expression in the respective groups are indicated in each histogram. 

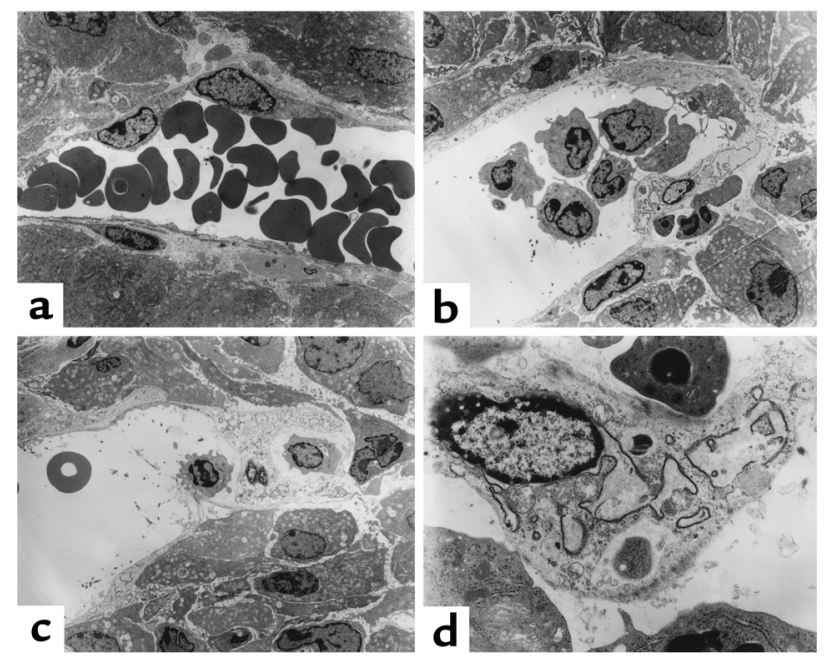

Figure 9

Impact of administration of IL-12/pulse IL-2 on the ultrastructural histology of endothelial cells within established Renca tumors. Intrarenal Renca tumors were resected from mice treated with IL-12 ( $0.5 \mu \mathrm{g}$ daily on days $11-14)$, pulse IL-2 (300,000 IU twice daily on day 11), or vehicle alone, and tissue specimens were processed for subsequent examination using electron microscopy. (a) Endothelial cells line a blood vessel within a control Renca tumor. $\times 6,000$. (b-d) Endothelial cells from Renca tumors treated with IL-12/pulse IL-2. (b and c) $\times 6000$. (d) $\times 30,000$.

potential mediators of apoptosis, within the local tumor site of Renca-bearing WT but not $I F N-\gamma^{-1}$ knockout mice (Figure 7). Additional studies in WT mice demonstrated that IL-12/pulse IL-2 administration had no impact on the constitutive expression of various proangiogenic mediators including VEGF, angiogenin, angiopoietin- 1 , TGF- $\beta_{2}$, FLT-1, FLK-1, and TIE-2 within primary Renca tumors (data not shown). These results demonstrated that administration of IL$12 /$ pulse IL-2 rapidly altered the expression of genes encoding both inhibitors of angiogenesis and inducers of apoptosis in an IFN- $\gamma$-dependent manner within the local tumor site.

Modulation of lymphocyte FasL expression. The central role of $\mathrm{CD}^{+} \mathrm{T}$ cells in mediating the antitumor activity of IL-12/pulse IL-2, and the ability of this combination to induce infiltration of $\mathrm{CD}^{+} \mathrm{T}$ cells and upregulate the expression of both Fas and FasL genes in the local tumor site, implied that the treatment could be upregulating FasL on $T$ cells. This hypothesis is further supported by additional data showing that IL-12/pulse IL-2 enhanced expression of the gene encoding FasL in the draining lymph nodes of mice bearing established subcutaneous Renca tumors (data not shown). In fact, the combination of IL-12 and IL-2 synergistically enhanced cell surface expression of FasL as assessed by flow cytometry on activated lymph node-derived lymphocytes in vitro (Figure 8 ). The proportions of cells expressing FasL were as follows: control $=3 \%$; IL-2 $=6 \%$; IL-12 $=15 \%$; IL-12/IL-2 = 53\%. Subsequent analyses found that under such conditions virtually $100 \%$ of these cells are $\mathrm{T}$ cells and that the observed increases in cell surface FasL expression occur on both $\mathrm{CD}^{+}$(control $=3 \%$; IL-2 $=30 \%$; IL-12 = 12\%; IL-12/IL-2 = 62\%) and $\mathrm{CD}^{+} \mathrm{T}$ lymphocytes (control $=0 \%$; IL-2 $=28 \%$; IL-12 = 23\%; IL-12/IL-2 = 70\%) (data not shown).

Administration of IL-12/pulse IL-2 induces Fas-dependent endothelial cell apoptosis in established renal tumors. The gene expression data presented above (Figure 7), combined with the antineovascular effects detailed in Figures 4-6, demonstrate an IFN- $\gamma$-dependent shift in the profile of antiangiogenic and proapoptotic gene expression in conjunction with a grossly evident antiangiogenic effect in mice treated with IL-12/pulse IL-2. However, the results do not provide insight into whether the observed antiangiogenic effects are limited to an ability to inhibit the formation of neovascularization or might also include the destruction of existing vasculature (i.e., via induction of endothelial cell apoptosis). To more definitively investigate this question, electron microscopy was used to study ultrastructural changes in intrarenal tumor implants early (within 4 days) in the course of therapy with IL12/pulse IL-2. Although endothelial cell populations from control intrarenal tumors possess normal ultrastructural histology (Figure 9a), endothelial cells in tumors from mice treated with IL-12/IL-2 were easily observed to be injured, as defined by swelling of the endoplasmic reticulum, nuclear condensation and fragmentation, and in some instances frank cellular destruction (Figure 9, c-d). In addition, many tumor cells from mice treated with IL-12/pulse IL-2 exhibit nuclear condensation and fragmentation, cytoplasmic vascularization, and in some instances, overt cellular degeneration with cellular ghosts (not shown).

In light of the rapid induction of endothelial cell apoptosis within established Renca tumors after treatment with IL-12/IL-2 and the observed IFN$\gamma$-dependent enhancement of both Fas and FasL gene expression in the tumor microenvironment, we next investigated the role of the Fas/FasL pathway in mediating early IL-12/IL-2-induced endothelial injury. We again used electron microscopy to compare the ultrastructural histology of endothelial cells from WT control mice to those from WT or lpr mice (with dysfunctional Fas) treated with IL-12/pulse IL-2. As shown in Figure 9, endothelial cells from WT mice treated with IL-12/pulse IL-2 demonstrate retraction and severe nuclear condensation and/or fragmentation (Figure 10, d-f) compared with tumorassociated endothelial cells from WT control mice (Figure 10, a-c). Although a small subset of endothelial cells from tumors implanted in lpr mice with dysfunctional Fas demonstrate some retraction, severe ultrastructural changes consistent with apoptosis were not observed (Figure 10, g-i). Collectively, these findings demonstrate that administration of IL12/pulse IL-2 induces rapid ultrastructural changes consistent with tumor and/or endothelial apoptosis and that apoptosis of tumor-associated endothelial 

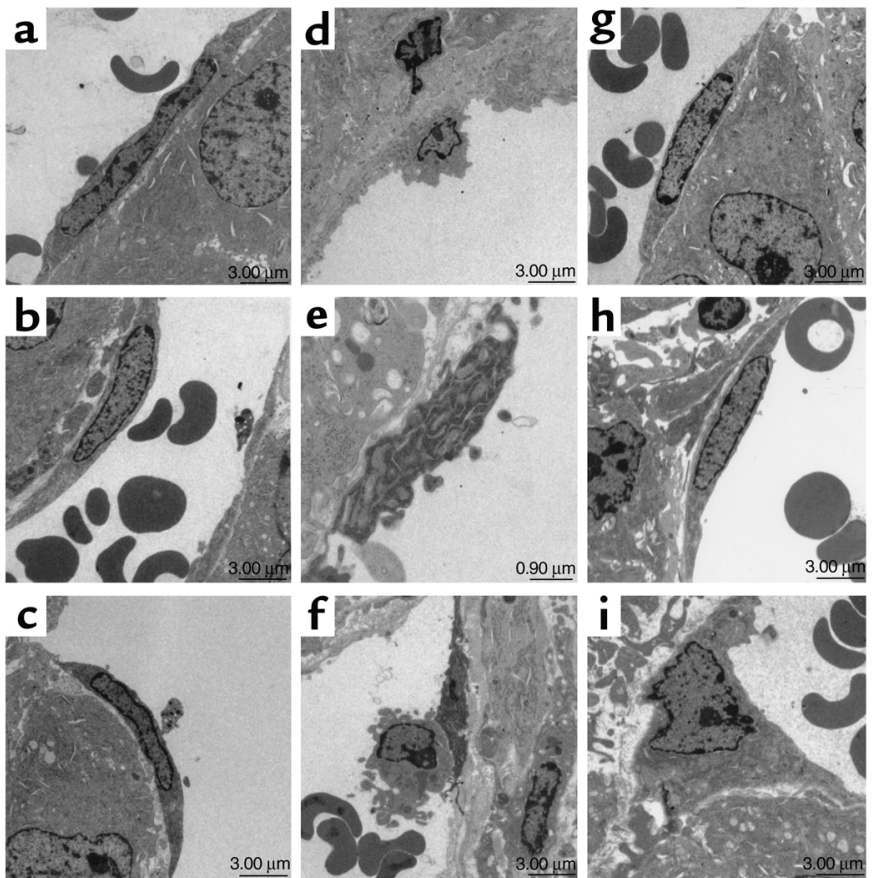

\section{Figure 10}

Role of the Fas/FasL pathway in IL-12/pulse IL-2-induced endothelial apoptosis within established renal tumors. Intrarenal tumors were resected from WT or Ipr mice treated with IL-12 (0.5 $\mu \mathrm{g}$ daily on days 11-14), pulse IL-2 (300,000 IU twice daily on day 11), or vehicle alone, as indicated, and tissue specimens were processed for subsequent examination using electron microscopy. (a-c) Endothelial cells from control Renca tumors. (d-f) Endothelial cells from Renca tumors in IL-12/pulse IL-2-treated WT and $(\mathbf{g}-\mathbf{i})$ Ipr mice. $(\mathbf{a}-\mathbf{d}, \mathbf{f}-\mathbf{i}) \times 3,000$. (e) $\times 9,000$.

cells after treatment with IL-12/pulse IL-2 is dependent on a functional Fas/FasL pathway.

The antitumor activity of IL-12/pulse IL-2 is dependent on FasL. The synergistic enhancement of FasL expression observed on $\mathrm{CD}^{+} \mathrm{T}$ lymphocytes treated with IL-12 plus IL-2 in vitro and the ability of this combination to potently upregulate Fas/FasL gene expression and rapidly induce Fas-dependent endothelial cell apoptosis within the local tumor site strongly suggest that the Fas/FasL pathway is critical for the overall tumor regression induced by IL-12/pulse IL-2. This hypothesis was tested by comparing the antitumor activity of IL-12/pulse IL-2 in Renca-bearing WT versus gld mice that express a dysfunctional FasL gene (Figure 11). Treatment of WT mice bearing metastatic Renca significantly $(P=0.0039)$ increased the incidence of complete responses to $64 \%$ (7/11) as compared with $0 \%$ WT or gld control mice treated with vehicle alone or gld mice treated with IL-12/pulse IL-2. These results show that the therapeutic effects of IL-12/pulse IL-2 are largely lost in mice that express dysfunctional FasL.

\section{Discussion}

Individual cytokines such as IL-2, which is approved for the treatment of patients with advanced melanoma and renal cell carcinoma, have found specific therapeutic niches in the treatment of human cancer. However, the nature of the biological responses, and therefore the actual therapeutic benefits of individual cytokines, have been modest. Because immune responses are regulated by a complex cytokine network, it is likely that appropriate combinations of cytokines with complementary mechanisms of action will ultimately provide the most effective means for therapeutic enhancement of the host antitumor immune response. IL-2 and IL-12 are two potent type 1 cytokines that activate leukocytes by binding to distinct receptors $(23,24)$ and subsequent use of parallel intracellular signaling pathways (25). Although these two cytokines possess synergistic immunoregulatory $(1,4-6)$ and antitumor (8-12) activities, relatively little is known about specific mechanisms accounting for the therapeutic synergy between IL-12 and IL- 2 or the degree to which classical innate or adaptive immune responses and other antitumor mechanisms may converge to effect a therapeutic antitumor response in vivo.

As suggested from our previous studies (12), the antitumor activity of IL-12/pulse IL-2 is critically dependent on $\mathrm{T}$ cells, in particular the $\mathrm{CD}^{+}$subset, and administration of this cytokine combination markedly enhances the infiltration of $\mathrm{CD}^{+} \mathrm{T}$ cells into the local tumor site. Previous reports showing that the combination of IL-12 and IL-2 potently enhances IFN- $\gamma$ production by $\mathrm{T}$ and/or NK cells in vitro $(1,4,5)$, and the identification of IFN- $\gamma$ as a central mediator of the immunoregulatory (1) and antitumor $(2,3)$ effects of IL-12 alone suggested a role for IFN- $\gamma$ in the antitumor effects of IL12/pulse IL-2. Administration of IL-12/pulse IL-2 markedly enhances the production of IFN- $\gamma$ in Rencabearing mice, and the antitumor activity of this regimen is critically dependent on the induction of endogenous IFN- $\gamma$ production. We have demonstrated here that not only are $\mathrm{CD}^{+} \mathrm{T}$ cells and endogenous IFN- $\gamma$ production critical components of the antitumor activity of IL$12 /$ pulse IL-2, but also that a large percentage of $\mathrm{CD}^{+}$ $\mathrm{T}$ cells contribute to this IFN- $\gamma$ production in vivo. Nonetheless, the overall role of IFN- $\gamma$ is clearly complex. Although attempts to reproduce the antitumor effects of IL-12/pulse IL-2 with systemic administration of recombinant IFN- $\gamma$ protein via various routes and/or schedules have been unsuccessful (R.H. Wiltrout, unpublished observations), the maintenance of elevated systemic IFN- $\gamma$ levels in patients treated with IL-12 correlates with the induction of overt tumor regression (16). Furthermore, we have found that combined administration of IL-12/pulse IL-2 maintains systemic IFN- $\gamma$ levels in tumor-bearing mice more effectively than IL-2 or IL-12 alone (R.H. Wiltrout, unpublished observations). Collectively, these findings suggest that maintenance of threshold levels of IFN- $\gamma$ and/or other cofactors that work in concert with IFN- $\gamma$ may be vital for tumor regression induced by IL-12/pulse IL-2. 


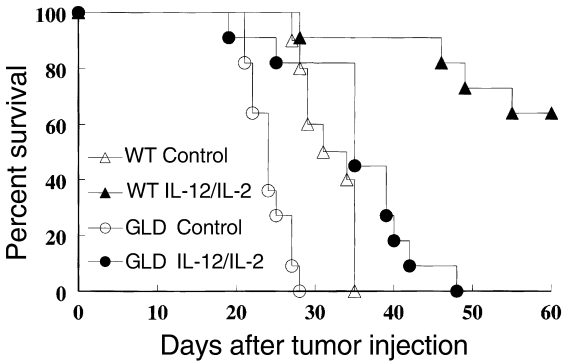

\section{Figure 11}

Role of FasL in mediating the antitumor activity of IL-12/pulse IL-2 against metastatic Renca. Cohorts of WT versus gld mice bearing intrarenal Renca tumor implants underwent unilateral nephrectomy to remove the primary tumor-bearing kidney on day 12 after tumor implantation and were subsequently treated with IL-12 $(0.5 \mu \mathrm{g}$ daily on days $13-16,20-24,27-31$, and 34-37), pulse IL-2 (300,000 IU twice daily on days $13,20,27$, and 34 ), or vehicle alone. Mice surviving at last follow-up were tumor-free.

We have observed substantial reductions in tumor vascularity and clearly evident foci of coagulative necrosis/apoptosis in histologic sections of tumors from mice treated with IL-12/pulse IL-2. In the present study, we have investigated molecular mechanisms accounting for these observed histologic changes and the role of endogenous IFN- $\gamma$ as a proximal inducer of these effects. IL-12 alone inhibits the vascularization of hydron pellets, Gelfoam sponges, or Matrigel plugs containing bFGF and/or tumor cells as an angiogenic stimulus $(15,22,26-28)$ and does so by IFN- $\gamma$-dependent mechanisms in several of these models of angiogenesis $(15,26,27)$. Using a novel latex-infusion technique, we have now demonstrated, we believe for the first time, that an IL-12-based therapy can induce substantial reductions in vascularization directly within orthotopically implanted tumors, and these effects are demonstrable after as little as one cycle of therapy with IL-12/pulse IL-2. Furthermore, we also have shown formally that these antineovascular effects are lost in $I F N-\gamma^{\prime-}$ mice, thus demonstrating a key role for induction of endogenous IFN- $\gamma$ production in both the antineovascular effects as well as in the overall antitumor activity of IL-12/pulse IL-2.

We subsequently investigated potential molecular pathways mediating the IFN- $\gamma$-dependent antineovascular and antitumor activity of IL-12/pulse IL-2, including the impact of this regimen on the expression of genes encoding relevant modulators of angiogenesis, as well as potential mediators of tumor and/or endothelial apoptosis within the local tumor site. IL-12/pulse IL-2 potently enhances expression of genes encoding IP-10 and Mig, two CXC-chemokines with both antiangiogenic and $\mathrm{T}$ cell chemoattractant activity, and does so by an IFN- $\gamma$-dependent mechanism. Nonetheless, only a portion of the overall antitumor activity of IL12/pulse IL-2 can be attributed to induction of IP-10 and Mig. Concurrent $\mathrm{Ab}$ neutralization of IP-10 and/or Mig only partially attenuates the induction of complete tumor regression by IL-12/pulse IL-2, while administration of maximal doses of IP-10/Mig with or without IL-2 fails to reproduce the antitumor effects of systemic IL-12/pulse IL-2 (J.M. Wigginton and R. H. Wiltrout, unpublished observations). Furthermore, treatment of Renca-bearing WT mice with IL-12/pulse IL-2 does not appreciably alter the local expression of various proangiogenic mediators, including VEGF, FLT-1, FLK-1, angiopoietin-1, TIE- 2 , angiogenin, TGF- $\beta_{2}$, MMP- 2 , and MMP-9 (J.M. Wigginton and R.H. Wiltrout, unpublished observations). These observations suggest that other IFN- $\gamma$-inducible factors may play a prominent role in the antineovascular and/or overall antitumor effects of IL-12/pulse IL-2, as well.

Based on light microscopy observations demonstrating substantial areas of coagulative necrosis and/or apoptosis with nuclear debris in Renca tumors after treatment with IL-12/pulse IL-2 (J.M. Wigginton, unpublished observation) and previous reports of in vitro modulation of the Fas/FasL apoptosis pathway by IL-2 and/or IFN- $\gamma$ (29-32), we speculated that apoptosis and, more specifically, the Fas/FasL pathway, may contribute to the antiangiogenic and antitumor effects of IL-12/pulse IL-2. Fas is expressed by a range of normal, preneoplastic, and malignant cell types (29-31, 33, 34 ), while FasL is expressed in various immunologically privileged sites $(35,36)$ on cytolytic effector cell populations $(30,37)$ and on tumor cells $(38-40)$. The Fas/FasL apoptosis pathway has been implicated in the regulation of $\mathrm{T}$ cell homeostasis $(41,42)$, maintenance of local immune privilege $(35,36)$, and in the cytolytic activity of $T$ and/or NK cells $(37,43,44)$. In some models, downregulation of Fas expression occurs in conjunction with the transition of cells to a malignant phenotype $(38,45)$, while in others, tumor cell FasL expression and the induction of Fas-mediated apoptosis of infiltrating effector cells has been implicated as a mechanism of tumor self-defense and endogenous immunosuppression (38-40). Thus, it appears that the Fas/FasL interaction may either promote or limit neoplastic progression and tumor growth depending on the model system under consideration.

In our studies, IL-12/pulse IL-2 inhibits neovascularization and enhances both Fas and FasL gene expression within the local tumor site by an IFN$\gamma$-dependent mechanism and also induces FasL gene expression within the draining lymph nodes of tumorbearing mice. This combination also synergistically enhances cell surface expression of FasL on lymph node-derived $\mathrm{CD}^{+} \mathrm{T}$ cells, potently upregulates $\mathrm{CD} 8^{+}$ T cell IFN- $\gamma$ expression in vivo, and induces the infiltration of $\mathrm{CD}^{+} \mathrm{T}$ cells into the local tumor site, further implicating interactions between $\mathrm{CD}^{+} \mathrm{T}$ cells, IFN- $\gamma$, and the Fas/FasL pathway in the antitumor mechanisms engaged by IL-12/pulse IL-2. Based on the observed histologic changes and enhanced Fas/FasL gene expression in the local tumor site of mice treated with IL-12/pulse IL-2, we investigated whether apoptosis was induced in these tumors by 
using transmission electron microscopy. Ultrastructural changes consistent with apoptosis were readily detectable in endothelial cell populations after only one cycle of therapy with IL-12/pulse IL-2, including nuclear condensation and/or fragmentation, swelling of the endoplasmic reticulum, and global cellular swelling and/or overt cellular degeneration. Collectively, these observations suggested that administration of IL-12/pulse IL-2 could disrupt tumor neovascularization by IFN- $\gamma$-dependent mechanisms that include not only inhibition of vessel establishment (i.e., by the induction of antiangiogenic chemokines such as IP-10/Mig), but also induction of vascular endothelial injury with destruction of existing vasculature as well, possibly by Fas/FasL-mediated apoptosis. Large areas of apoptotic tumor cells with nuclear condensation and/or fragmentation, cytoplasmic vacuolization, and in extreme instances, cellular ghosts with plasma membranes containing degenerated cytoplasmic and nuclear components, also were evident in the tumor bed of treated mice. Notably, although Renca does not express FasL, treatment with IFN- $\gamma$ and/or TNF- $\alpha$ enhances cell surface Fas expression and the susceptibility of Renca to Fas-mediated killing upon subsequent exposure to anti-Fas Ab's in vitro (34). To directly address the role of the Fas/FasL pathway in the antiangiogenic activity of IL-12/pulse IL-2, we used electron microscopy to compare the ultrastructural histology of tumor-associated endothelial cells from WT control mice to those from WT or lpr (with dysfunctional Fas) mice treated with IL-12/IL-2. These studies have demonstrated that the ability of IL12/pulse IL-2 to induce endothelial cell injury and apoptosis is markedly reduced in lpr mice with dysfunctional Fas compared with WT mice, thus demonstrating a role for the Fas/FasL pathway in the antiangiogenic activity of IL-12/pulse IL-2.

Furthermore, we found that although administration of IL-12/pulse IL-2 could prolong the survival of tumorbearing gld mice with dysfunctional FasL, the ability of this regimen to induce complete tumor regression is ablated in these mice. Previous studies using systemic IL-12 or IL-12 gene therapy alone have failed to demonstrate a role for Fas/FasL in the antitumor effects of IL-12 $(46,47)$, although IL-12 can increase the frequency of a CD3 ${ }^{+}, \mathrm{CD}^{-}, \mathrm{CD}^{-} \mathrm{B} 220^{+}$lymphoid population capable of lysing Fas-positive tumor cells in vitro (48). Thus, the present studies also provide the first definitive evidence of a role for the Fas/FasL pathway in mediating the overall antitumor activity of IL-12-based therapy (i.e., IL-12/pulse IL-2) in vivo.

Overall, the studies described in this report provide new insight into the novel convergence of host antitumor mechanisms that may be engaged by biological therapies such as IL-12/pulse IL-2. These mechanisms include not only conventional aspects of the host immune response, but also downstream engagement of other mechanisms, including inhibition of tumor neovascularization and the induction of vascular injury with endothelial cell apoptosis. Our results are consistent with the hypothesis that treatment with IL$12 /$ pulse IL-2 results in an interaction of infiltrating $\mathrm{CD}^{+} \mathrm{FasL}^{+} \mathrm{T}$ lymphocytes with $\mathrm{Fas}^{+}$endothelial and/or tumor cells in the tumor microenvironment and that this interaction induces vascular injury with endothelial and/or tumor apoptosis and subsequent tumor regression. Additionally, these studies emphasize the complex role of IFN- $\gamma$ and its interactions with the Fas/FasL pathway in mediating the therapeutic effects of IL-12/pulse IL-2 and provide several clear biological endpoints for evaluation during a phase I clinical investigation of this combination, which we have initiated here in the National Cancer Institute.

\section{Acknowledgments}

The authors thank Joyce Vincent and Susan Charbonneau for editing and manuscript preparation, Kirk Volker for technical assistance, Gregory Alvord for statistical analysis, and Maria Tsokos for helpful discussions. The generous support of the Children's Cancer Foundation (to J.M. Wigginton) is also gratefully acknowledged. This project has been funded in whole or in part with federal funds from the National Cancer Institute, the NIH, under contract N01-CO-56000. The content of this publication does not necessarily reflect the views or policies of the Department of Health and Human Services, nor does mention of trade names, commercial products, or organizations imply endorsement by the US Government. The publisher or recipient acknowledges the right of the US Government to retain a nonexclusive, royalty-free license in and to any copyright covering this article.

1. Trinchieri, G. 1998. Interleukin-12: a cytokine at the interface of inflammation and immunity. Adv. Immunol. 70:83-243.

2. Brunda, M.J., et al. 1993. Antitumor and antimetastatic activity of interleukin 12 against murine tumors. J. Exp. Med. 178:1223-1230.

3. Nastala, C.L., et al. 1994. Recombinant IL-12 administration induces tumor regression in association with IFN-gamma production. $J$. Immunol. 153:1697-1706.

4. Gately, M.K., Wilson, D.E., and Wong, H.L. 1986. Synergy between recombinant interleukin 2 (rIL 2) and IL 2-depleted lymphokine-containing supernatants in facilitating allogeneic human cytolytic T lymphocyte responses in vitro. J. Immunol. 136:1274-1282.

5. Chan, S.H., et al. 1991. Induction of interferon gamma production by natural killer cell stimulatory factor: characterization of the responder cells and synergy with other inducers. J. Exp. Med. 173:869-879.

6. Perussia, B., et al. 1992. Natural killer (NK) cell stimulatory factor or IL12 has differential effects on the proliferation of TCR-alpha beta+, TCRgamma delta+ T lymphocytes, and NK cells. J. Immunol. 149:3495-3502.

7. Wigginton, J.M., et al. 1996. Interleukin 12 primes macrophages for nitric oxide production in vivo and restores depressed nitric oxide production by macrophages from tumor-bearing mice: implications for the antitumor activity of interleukin 12 and/or interleukin 2. Cancer Res. 56:1131-1136

8. Rossi, A.R., Pericle, F., Rashleigh, S., Janiec, J., and Djeu, J.Y. 1994. Lysis of neuroblastoma cell lines by human natural killer cells activated by interleukin-2 and interleukin-12. Blood. 83:1323-1328.

9. Rashleigh, S.P., Kusher, D.I., Endicott, J.N., Rossi, A.R., and Djeu, J.Y. 1996. Interleukins 2 and 12 activate natural killer cytolytic responses of peripheral blood mononuclear cells from patients with head and neck squamous cell carcinoma. Arch. Otolaryngol. Head Neck Surg. 122:541-547.

10. Zitvogel, L., et al. 1995. Cancer immunotherapy of established tumors with IL-12. Effective delivery by genetically engineered fibroblasts. $J$. Immunol. 155:1393-1403.

11. Vagliani, M., et al. 1996. Interleukin 12 potentiates the curative effect of a vaccine based on interleukin 2-transduced tumor cells. Cancer Res. 56:467-470. 
12. Wigginton, J.M., et al. 1996. Administration of interleukin 12 with pulse interleukin 2 and the rapid and complete eradication of murine renal carcinoma. J. Natl. Cancer Inst. 88:38-43.

13. Wigginton, J.M., et al. 2001. Complete regression of established spontaneous mammary carcinoma and the therapeutic prevention of genetically programmed neoplastic transition by IL-12/pulse IL-2: induction of local T cell infiltration, Fas/Fas ligand gene expression, and mammary epithelial apoptosis. J. Immunol. 166:1156-1168.

14. Trinchieri, G. 1994. Interleukin-12: a cytokine produced by antigen-presenting cells with immunoregulatory functions in the generation of Thelper cells type 1 and cytotoxic lymphocytes. Blood. 84:4008-4027.

15. Coughlin, C.M., et al. 1998. Tumor cell responses to IFNgamma affect tumorigenicity and response to IL-12 therapy and antiangiogenesis. Immunity. 9:25-34.

16. Gollob, J.A., et al. 2000. Phase I trial of twice-weekly intravenous interleukin 12 in patients with metastatic renal cell cancer or malignant melanoma: ability to maintain IFN-gamma induction is associated with clinical response. Clin. Cancer Res. 6:1678-1692.

17. Laszlo, J., et al. 1990. Phase I studies of recombinant interferon-gamma. J. Biol. Response Mod. 9:185-193.

18. Huben, R.P., Connelly, R., Goldrosen, M.H., Murphy, G.P., and Pontes, J.E. 1983. Immunotherapy of a murine renal cancer. J. Urol. 129:1075-1078.

19. Rosenberg, S.A., et al. 1984. Biological activity of recombinant human interleukin-2 produced in Escherichia coli. Science. 223:1412-1414.

20. Wang, A., Lu, S.D., and Mark, D.F. 1984. Site-specific mutagenesis of the human interleukin-2 gene: structure-function analysis of the cysteine residues. Science. 224:1431-1433.

21. Park, J.W., et al. 2001. Primary hepatocytes from mice treated with IL2/IL-12 produce T cell chemoattractant activity that is dependent on monokine induced by IFN-gamma (Mig) and chemokine responsive to gamma-2 (Crg-2). J. Immunol. 166:3763-3770.

22. Watanabe, M., et al. 1997. Regulation of local host-mediated anti-tumor mechanisms by cytokines: direct and indirect effects on leukocyte recruitment and angiogenesis. Am. J. Pathol. 150:1869-1880.

23. Desai, B.B., et al. 1992. IL-12 receptor. II. Distribution and regulation of receptor expression. J. Immunol. 148:3125-3132.

24. Nelson, B.H., and Willerford, D.M. 1998. Biology of the interleukin-2 receptor. Adv. Immunol. 70:1-81.

25. Bacon, C.M., et al. 1995. Interleukin 12 (IL-12) induces tyrosine phosphorylation of JAK2 and TYK2: differential use of Janus family tyrosine kinases by IL-2 and IL-12. J. Exp. Med. 181:399-404.

26. Voest, E.E., et al. 1995. Inhibition of angiogenesis in vivo by interleukin 12. J. Natl. Cancer Inst. 87:581-586.

27. Sgadari, C., Angiolillo, A.L., and Tosato, G. 1996. Inhibition of angiogenesis by interleukin-12 is mediated by the interferon-inducible protein 10. Blood. 87:3877-3882.

28. Coughlin, C.M., et al. 1998. Interleukin-12 and interleukin-18 synergistically induce murine tumor regression which involves inhibition of angiogenesis. J. Clin. Immunol. 101:1441-1452.

29. Leithauser, F., et al. 1993. Constitutive and induced expression of APO-
1 , a new member of the nerve growth factor/tumor necrosis factor receptor superfamily, in normal and neoplastic cells. Lab. Invest. 69:415-429.

30. Suda, T., et al. 1995. Expression of the Fas ligand in cells of T cell lineage. J. Immunol. 154:3806-3813.

31. Weller, M., et al. 1994. Anti-Fas/APO-1 antibody-mediated apoptosis of cultured human glioma cells. Induction and modulation of sensitivity by cytokines. J. Clin. Invest. 94:954-964.

32. Komada, Y., et al. 1997. Fas/APO-1 (CD95)-mediated cytotoxicity is responsible for the apoptotic cell death of leukaemic cells induced by interleukin-2-activated T cells. Br. J. Haematol. 96:147-157.

33. Shen, K., and Novak, R.F. 1997. Fas-signaling and effects on receptor tyrosine kinase signal transduction in human breast epithelial cells. Biochem. Biophys. Res. Commun. 230:89-93.

34. Lee, J.K., et al. 2000. IFN- $\gamma$-dependent delay of in vivo tumor progression by Fas-overexpression on murine renal cancer cells. J. Immunol. 164:231-239.

35. Bellgrau, D., et al. 1995. A role for CD95 ligand in preventing graft rejection. Nature. 377:630-632.

36. Griffith, T.S., Brunner, T., Fletcher, S.M., Green, D.R., and Ferguson, T.A 1995. Fas ligand-induced apoptosis as a mechanism of immune privilege. Science. 270:1189-1192.

37. Arase, H., Arase, N., and Saito, T. 1995. Fas-mediated cytotoxicity by freshly isolated natural killer cells. J. Exp. Med. 181:1235-1238.

38. Strand, S., et al. 1996. Lymphocyte apoptosis induced by CD95 (APO$1 /$ Fas) ligand-expressing tumor cells: a mechanism of immune evasion? Nat. Med. 2:1361-1366.

39. Hahne, M., et al. 1996. Melanoma cell expression of Fas(Apo-1/CD95) ligand: implications for tumor immune escape. Science. 274:1363-1366.

40. O'Connell, J., O'Sullivan, G.C., Collins, J.K., and Shanahan, F. 1996. The Fas counterattack: Fas-mediated T cell killing by colon cancer cells expressing Fas ligand. J. Exp. Med. 184:1075-1082.

41. Watanabe-Fukunaga, R., Brannan, C.I., Copeland, N.G., Jenkins, N.A., and Nagata, S. 1992. Lymphoproliferation disorder in mice explained by defects in Fas antigen that mediates apoptosis. Nature. 356:314-317.

42. Takahashi, T., et al. 1994. Generalized lymphoproliferative disease in mice, caused by a point mutation in the Fas ligand. Cell. 76:969-976.

43. Hanabuchi, S., et al. 1994. Fas and its ligand in a general mechanism of T-cell-mediated cytotoxicity. Proc. Natl. Acad. Sci. USA. 91:4930-4934.

44. Kagi, D., et al. 1994. Fas and perforin pathways as major mechanisms of T cell-mediated cytotoxicity. Science. 265:528-530.

45. Keane, M.M., Ettenberg, S.A., Lowrey, G.A., Russell, E.K., and Lipkowitz, S. 1996. Fas expression and function in normal and malignant breast cell lines. Cancer Res. 56:4791-4798.

46. Hashimoto, W., et al. 1999. Differential antitumor effects of administration of recombinant IL-18 or recombinant IL-12 are mediated primarily by Fas-Fas ligand. J. Immunol. 163:583-589.

47. Kodama, T., et al. 1999. Perforin-dependent NK cell cytotoxicity is sufficient for anti-metastatic effect of IL-12. Eur. J. Immunol. 29:1390-1396.

48. Tsutsui, T., et al. 1997. Administration of IL-12 induces a CD3 ${ }^{+} \mathrm{CD} 4$ CD8- $3220^{+}$lymphoid population capable of eliciting cytolysis against Fas-positive tumor cells. J. Immunol. 159:2599-2605. 